\title{
On the geometric determination of the poles of Hodge and motivic zeta functions
}

\author{
B. Rodrigues*
}

October 23, 2003

\begin{abstract}
To any polynomial $f \in \mathbb{C}\left[x_{1}, \ldots, x_{n}\right] \backslash \mathbb{C}$ one can associate the zeta function $Z_{\text {Hod }}(f, s)$ on the level of Hodge polynomials. This function is a specialization of the well-known motivic zeta function. For $n=2$ Veys gave a complete geometric determination of the poles of $Z_{\mathrm{Hod}}(f, s)$. In this paper we work in arbitrary dimension. We mainly provide for a given rational number a geometric sufficient condition for being a pole of $Z_{\text {Hod }}(f, s)$. The same statement also holds for the motivic zeta function. Moreover, our results even remain true when we consider the zeta functions associated to an effective $\mathbb{Q}$-Cartier divisor $D$ on a $\mathbb{Q}$-Gorenstein variety with singular locus contained in $\operatorname{Supp} D$.
\end{abstract}

\section{Introduction}

(0.1) Let $f \in \mathbb{C}[x, y] \backslash \mathbb{C}$ with $f(0)=0$ and let $h: X \rightarrow \mathbb{A}_{\mathbb{C}}^{2}$ be an embedded resolution of $f^{-1}\{0\}$ in the germ $\left(\mathbb{A}_{\mathbb{C}}^{2}, 0\right)$ of $\mathbb{A}_{\mathbb{C}}^{2}$ at the origin, constructed by successive blowing-ups. Denote by $E_{i}, i \in T=T_{e} \cup T_{s}$, the irreducible components of $h^{-1}\left(f^{-1}\{0\}\right)$, where $E_{i}$ is an irreducible component of the exceptional divisor for $i \in T_{e}$ and of the strict transform of $f^{-1}\{0\}$ in $X$ for $i \in T_{s}$. For each $i \in T$ we denote by $N_{i}$ and $\nu_{i}-1$ the multiplicities of $E_{i}$ in the divisor on $X$ of $f \circ h$ and $h^{*}(d x \wedge d y)$, respectively. To $f$ one associates the zeta function

$$
\begin{aligned}
Z_{\mathrm{Hod}}(f, s):= & \sum_{i \in T_{e}} H\left(E_{i}^{\circ}\right) \frac{u v-1}{(u v)^{\nu_{i}+s N_{i}}-1}+ \\
& \sum_{\{i, j\} \subseteq T} H\left(E_{i} \cap E_{j}\right) \frac{(u v-1)^{2}}{\left((u v)^{\nu_{i}+s N_{i}}-1\right)\left((u v)^{\nu_{j}+s N_{j}}-1\right)},
\end{aligned}
$$

where $E_{i}^{\circ}:=E_{i} \backslash \bigcup_{j \neq i} E_{j}$ and $H(\cdot)$ denotes the Hodge polynomial. For each variety $V$ this Hodge polynomial $H(V)$ is a polynomial over $\mathbb{Z}$ in two variables $u$ and $v$, see (1.3). We consider the expression above as a rational function in the variable $T=(u v)^{-s}$ and one can prove that it does not depend on the chosen resolution, see also (1.3).

*Postdoctoral Fellow of the Fund for Scientific Research - Flanders (Belgium).

2000 Mathematics Subject Classificaton. 14B05 14E15 14J17 (32S45) 
(0.2) We are interested in the poles of $Z_{\mathrm{Hod}}(f, s)$. Here we say that a rational number $q$ is a pole if $(u v)^{-q}$ is, considering $Z_{\mathrm{Hod}}(f, s)$ as a rational function in the variable $T=(u v)^{-s}$. The expression for $Z_{\text {Hod }}(f, s)$ in $(0.1)$ in terms of an embedded resolution yields a list of the possible poles of $Z_{\text {Hod }}(f, s)$; namely $\left\{-\frac{\nu_{i}}{N_{i}} \mid i \in T\right\}$. We call the elements of this set the candidate poles. Now it is striking that very often a lot of these candidate poles are actually not really a pole. We want to decide geometrically whether or not a given candidate pole $-\frac{\nu_{i}}{N_{i}}, i \in T$, is a pole. In this case of two variables Veys proved the following theorem, which gives a complete geometric determination of the poles.

(0.2.1) Theorem ([13, Theorem 4.3]). Let $h$ be the minimal embedded resolution of $f^{-1}\{0\}$ in $\left(\mathbb{A}_{\mathbb{C}}^{2}, 0\right)$. Then a rational number $s_{\circ}$ is a pole of $Z_{\mathrm{Hod}}(f, s)$ if and only if $s_{\circ}=-\frac{1}{N_{i}}$ for some irreducible component $E_{i}$ of the strict transform of $f^{-1}\{0\}$ or $s_{\circ}=-\frac{\nu_{i}}{N_{i}}$ for some exceptional curve $E_{i}$ intersecting other components at least three times.

In fact Veys proved this result for the so-called topological zeta function, see for example (1.3) for the definition of this function; but it only needs a trivial verification to see that this immediately implies Theorem 0.2.1. The theorem also holds for an arbitrary embedded resolution, at least when we replace the condition "intersecting other components at least three times" by "intersecting other components $E_{j}$ with $\nu_{j}-\frac{\nu_{i}}{N_{i}} N_{j} \neq 1$ at least three times". Note that Theorem 0.2.1 actually gives a statement about the poles of order 1 and 2. In [9] we proved a generalization for arbitrary dimensions, i.e., for $f \in \mathbb{C}\left[x_{1}, \ldots, x_{n}\right]$, concerning the poles of order $n-1$ and $n$.

As an application of the techniques of the present paper we will give another proof of Theorem 0.2.1 in Section 4.

(0.2.2) The absolute value $\min _{i \in T}\left\{\nu_{i} / N_{i}\right\}$ of the largest candidate pole is in fact just the $\log$ canonical threshold of $f$ at 0 (and is thus independent of the chosen resolution), see [6, Section 8]. The whole set $\left\{-\frac{\nu_{i}}{N_{i}} \mid i \in T\right\}$ is of course not an invariant of $f$, but its subset consisting of the poles of $Z_{\text {Hod }}(f, s)$ is. Philosophically the poles of these zeta functions are induced by "important" components $E_{i}$, which occur in every resolution.

These remarks are also true in arbitrary dimension.

(0.3) In this paper we mainly look what happens when we proceed to the situation of higher dimensions; in particular we are interested in generalizations of Theorem 0.2.1. The notations for arbitrary dimensions will be very similar to those introduced in $(0.1)$ for the case of two variables, see Section 1 . To any polynomial $f \in \mathbb{C}\left[x_{1}, \ldots, x_{n}\right] \backslash \mathbb{C}$ with $f(0)=0$ we associate the zeta function

$$
Z_{\mathrm{Hod}}(f, s):=\sum_{I \subseteq T} H\left(E_{I}^{\circ} \cap h^{-1}\{0\}\right) \prod_{i \in I} \frac{u v-1}{(u v)^{\nu_{i}+s N_{i}}-1},
$$

given in terms of an embedded resolution $h: X \rightarrow \mathbb{A}_{\mathbb{C}}^{n}$ of $f^{-1}\{0\}$ in $\mathbb{A}_{\mathbb{C}}^{n}$ and where $E_{I}:=$ $\bigcap_{i \in I} E_{i}$ and $E_{I}^{\circ}:=E_{I} \backslash \bigcup_{j \in T \backslash I} E_{j}$.

(0.4) Fix an exceptional variety $E$ with $h(E)=\{0\}$ and suppose that none of the intersecting varieties $E_{i}, i \in T$, induces the same candidate pole as $E$. We denote by $\mathcal{R}$ the contribution of $E$ to the residue of $Z_{\mathrm{Hod}}(f, s)$ at $-\frac{\nu}{N}$. Of course, by the contribution of $E$, being equal to $E_{j}$ for some $j \in T$, to the residue of $Z_{\mathrm{Hod}}(f, s)$ at $-\frac{\nu}{N}$ we mean the residue at $-\frac{\nu}{N}$ of 
the function obtained from $Z_{\text {Hod }}(f, s)$ by restricting its defining expression, see (0.3), to the terms which correspond to the subsets $I$ of $T$ containing $j$. One easily obtains the following explicit formula for $\mathcal{R}$ :

$$
\mathcal{R}=\frac{1-u v}{N(u v)^{-\nu / N}}\left(\sum_{j \in I \subseteq T} H\left(E_{I}^{\circ}\right) \prod_{i \in I \backslash\{j\}} \frac{u v-1}{(u v)^{\nu_{i}-(\nu / N) N_{i}}-1}\right) .
$$

We want to find a geometric sufficient condition for $\mathcal{R}$ to be nonzero. In a previous paper [10] we were already able to prove (for $n=3$ ) the following result. Till now nothing more was known (for $n \geq 3$ ) about this non-vanishing question.

(0.4.1) Theorem. Let $f \in \mathbb{C}\left[x_{1}, x_{2}, x_{3}\right] \backslash \mathbb{C}$ and fix an embedded resolution $h: X \rightarrow \mathbb{A}_{\mathbb{C}}^{3}$ of $f^{-1}\{0\}$ in $\mathbb{A}_{\mathbb{C}}^{3}$. Let $E$ be a non-rational exceptional surface with $h(E)=\{0\}$ and numerical data $(\nu, N)$. Let $E_{i}, i=1, \ldots, r$, be the irreducible components of $h^{-1}\left(f^{-1}\{0\}\right)$, different from $E$, which intersect $E$. Suppose that $\nu_{i}-\frac{\nu}{N} N_{i} \neq 0,1$ for each $i \in\{1, \ldots, r\}$. If $\bar{\kappa}\left(E^{\circ}\right)=2$, then $\mathcal{R}$ is nonzero.

For this theorem we needed some extra conditions, namely the fact that $E$ has to be nonrational and that $\alpha_{i} \neq 1$ for each $i \in\{1, \ldots, r\}$. Moreover, the proof was quite long and required a lot of calculations. All this will be in sharp contrast with the generality of the results of the present paper and the brevity and elegance of their proofs. Here the main theorem is the following.

(0.4.2) Theorem. Let $f \in \mathbb{C}\left[x_{1}, \ldots, x_{n}\right] \backslash \mathbb{C}$ and fix an embedded resolution $h: X \rightarrow \mathbb{A}_{\mathbb{C}}^{n}$ of $f^{-1}\{0\}$ in $\mathbb{A}_{\mathbb{C}}^{n}$. Let $E$ be an exceptional variety with $h(E)=\{0\}$ and numerical data $(\nu, N)$. Let $E_{i}, i=1, \ldots, r$, be the irreducible components of $h^{-1}\left(f^{-1}\{0\}\right)$, different from $E$, which intersect $E$. Suppose that $\alpha_{i}:=\nu_{i}-\frac{\nu}{N} N_{i} \neq 0$ for each $i \in\{1, \ldots, r\}$. Then we have

$$
\left(\sum_{I \subseteq S} \frac{\chi\left(\left(E \cap E_{I}\right)^{\circ}\right)}{\prod_{i \in I} \alpha_{i}} \neq 0\right) \Rightarrow(\mathcal{R} \neq 0) \text {. }
$$

Here we denote by $S$ the set $\left\{i \in\{1, \ldots, r\} \mid \alpha_{i} \in \mathbb{Z}\right\}$.

Note that when $S=\emptyset$, the theorem yields the implication

$$
\left(\chi\left(E^{\circ}\right) \neq 0\right) \Rightarrow(\mathcal{R} \neq 0) .
$$

Even only this particular case of Theorem 0.4.2 gives us a very nice result, especially when one takes into account the generality it is stated in. As it will turn out, the technique of the present paper will also make it possible to add up several contributions to the residue at a candidate pole, to give similar results for the motivic zeta function and even for the poles of higher order. Moreover, all these facts will remain true when we consider the zeta functions associated to an effective $\mathbb{Q}$-Cartier divisor $D$ on a $\mathbb{Q}$-Gorenstein variety with singular locus contained in $\operatorname{Supp} D$, see [16] for their definition. We conclude by stating a remarkable corollary of our capability of adding several contributions.

(0.4.3) Theorem. Let $f \in \mathbb{C}\left[x_{1}, \ldots, x_{n}\right] \backslash \mathbb{C}$ and let $s_{\circ} \in \mathbb{Q}$. Fix an embedded resolution $h: X \rightarrow \mathbb{A}_{\mathbb{C}}^{n}$ of $f^{-1}\{0\}$ in $\mathbb{A}_{\mathbb{C}}^{n}$. Let $E_{j}, j=1, \ldots, k$, be precisely the irreducible components of $h^{-1}\left(f^{-1}\{0\}\right)$ satisfying $s_{\circ}=-\frac{\nu_{j}}{N_{j}}$. Let $E_{i}^{(j)}, i=1, \ldots, r_{j}$, be the irreducible components of 
$h^{-1}\left(f^{-1}\{0\}\right)$, different from $E_{j}$, which intersect $E_{j}$. Suppose that $\nu_{i}^{(j)}+s_{\circ} N_{i}^{(j)} \notin \mathbb{Z}$ for each $j \in\{1, \ldots, k\}$ and $i \in\left\{1, \ldots, r_{j}\right\}$ satisfying $E_{j} \cap E_{i}^{(j)} \cap h^{-1}\{0\} \neq \emptyset$.

If there exists an exceptional variety $E_{j}$ satisfying $j \in\{1, \ldots, k\}, h\left(E_{j}\right)=\{0\}$ and $\chi\left(E_{j}^{\circ}\right) \neq 0$, then $s_{\circ}$ is a pole of $Z_{\mathrm{Hod}}(f, s)$.

(0.5) In the first, rather short, section we give most of the necessary definitions and notations. In the second section we fix an exceptional variety $E$ and we find an appropriate condition under which the contribution of $E$ will certainly not vanish. In the next section we obtain a sufficient condition for a given rational number to be a pole. Then in Section 4 we make an excursion to the situation of curves, providing a new proof for Theorem 0.2.1.

\section{Preliminaries}

(1.1) Let $f$ be a nonconstant polynomial over $\mathbb{C}$ in $n$ variables, i.e., $f \in \mathbb{C}\left[x_{1}, \ldots, x_{n}\right] \backslash \mathbb{C}$. An embedded resolution of $f^{-1}\{0\}$ in $\mathbb{A}_{\mathbb{C}}^{n}$ consists of a nonsingular variety $X$ and a proper birational morphism $h: X \rightarrow \mathbb{A}_{\mathbb{C}}^{n}$ such that the restriction $h: X \backslash h^{-1}\left(f^{-1}\{0\}\right) \longrightarrow \mathbb{A}_{\mathbb{C}}^{n} \backslash f^{-1}\{0\}$ is an isomorphism and $h^{-1}\left(f^{-1}\{0\}\right)$ has only normal crossings in $X$. An embedded resolution always exists in characteristic zero by Hironaka [5].

A reduced closed subscheme $E$ of everywhere codimension one of a nonsingular variety $X$ is said to have only normal crossings, if for all $x \in X$ there exists a regular system of parameters $t_{1}, \ldots, t_{n}$ in the local ring $O_{X, x}$ of $X$ at $x$ such that the ideal in $O_{X, x}$ of each irreducible component of $E$ containing $x$ is generated by one of the $t_{i}$.

(1.2) Fix an embedded resolution $h: X \rightarrow \mathbb{A}_{\mathbb{C}}^{n}$ of $f^{-1}\{0\}$ in $\mathbb{A}_{\mathbb{C}}^{n}$ for a given polynomial $f \in \mathbb{C}\left[x_{1}, \ldots, x_{n}\right] \backslash \mathbb{C}$. Let $E_{i}, i \in T=T_{e} \cup T_{s}$, be the (reduced) irreducible components of $h^{-1}\left(f^{-1}\{0\}\right)$, where $E_{i}$ is a (reduced) irreducible component of the exceptional divisor for $i \in T_{e}$ and of the strict transform of $f^{-1}\{0\}$ in $X$ for $i \in T_{s}$. For each $i \in T$, let $N_{i}$ and $\nu_{i}-1$ be the multiplicities of $E_{i}$ in the divisor on $X$ of $f \circ h$ and $h^{*}\left(d x_{1} \wedge \cdots \wedge d x_{n}\right)$, respectively. The $\left(\nu_{i}, N_{i}\right)$, for $i \in T$, are called the numerical data of the resolution $(X, h)$. Although we denote by definition the irreducible components of $h^{-1}\left(f^{-1}\{0\}\right)$ by $E_{i}$, we will often denote them freely by $E, E_{i}^{(j)}$, et cetera. For the numerical data we then use the corresponding notation $(\nu, N),\left(\nu_{i}^{(j)}, N_{i}^{(j)}\right)$, et cetera. For $i \in T$ and $I \subseteq T$ we denote

$$
E_{i}^{\circ}:=E_{i} \backslash \bigcup_{j \neq i} E_{j}, \quad E_{I}:=\bigcap_{i \in I} E_{i} \quad \text { and } \quad E_{I}^{\circ}:=E_{I} \backslash \bigcup_{j \in T \backslash I} E_{j} .
$$

In particular when $I=\emptyset$, we have $E_{\emptyset}=X$. Remark that $X$ is the disjoint union of the $E_{I}^{\circ}$. We obviously have for a subset $I$ of $T$ either that $E_{I}=\emptyset$ or that each irreducible component of $E_{I}$ has dimension $n-|I|$.

(1.3) Let $f \in \mathbb{C}\left[x_{1}, \ldots, x_{n}\right] \backslash \mathbb{C}$ with $f(0)=0$ and let $h: X \rightarrow \mathbb{A}_{\mathbb{C}}^{n}$ be an embedded resolution of $f^{-1}\{0\}$ in $\mathbb{A}_{\mathbb{C}}^{n}$. The zeta function on the level of Hodge polynomials associated to $f$ is defined as

$$
Z_{\mathrm{Hod}}(f, s):=\sum_{I \subseteq T} H\left(E_{I}^{\circ} \cap h^{-1}\{0\}\right) \prod_{i \in I} \frac{u v-1}{(u v)^{\nu_{i}+s N_{i}}-1} \in \mathbb{Q}(u, v)(T),
$$


where we consider $(u v)^{-s}$ as a variable $T$ and where $H(\cdot)$ denotes the Hodge polynomial. For a variety $V$ this Hodge polynomial is given by $H(V):=\sum_{p, q} e^{p, q}(V) u^{p} v^{q} \in \mathbb{Z}[u, v]$; here $e^{p, q}(V)=\sum_{i \geq 0}(-1)^{i} h^{p, q}\left(H_{c}^{i}(V, \mathbb{C})\right)$ with $h^{p, q}\left(H_{c}^{i}(V, \mathbb{C})\right)$ the rank of the $(p, q)$-Hodge component of the $i-t h$ cohomology group with compact support of $V$.

When we replace $h^{-1}\{0\}$ by $X$ in the defining expression for $Z_{\mathrm{Hod}}(f, s)$, we obtain the definition of the global zeta function $Z_{\mathrm{Hod}, \mathrm{gl}}(f, s)$ on the level of Hodge polynomials.

Another interesting singularity invariant of $f^{-1}\{0\}$ is the topological zeta function [2]. It is defined as

$$
Z_{\mathrm{top}}(f, s):=\sum_{I \subseteq T} \chi\left(E_{I}^{\circ} \cap h^{-1}\{0\}\right) \prod_{i \in I} \frac{1}{\nu_{i}+s N_{i}} \in \mathbb{Q}(s),
$$

where $\chi(\cdot)$ denotes the topological Euler characteristic. Also here we have a global variant $Z_{\text {top,gl }}(f, s)$, obtained by replacing $h^{-1}\{0\}$ by $X$.

The remarkable fact that the defining expressions for $Z_{\mathrm{Hod}}(f, s)$ and $Z_{\mathrm{top}}(f, s)$ do not depend on the chosen resolution can be proved by obtaining them as a specialization of the motivic zeta function, wich is intrinsically defined by Denef and Loeser using motivic integration, see [3]. For the topological zeta function this fact of independency was originally proved in [2] by expressing it as a limit of Igusa's local zeta functions. The zeta function $Z_{\text {Hod }}(f, s)$ is a finer invariant than $Z_{\text {top }}(f, s)$; indeed, we can specialize $Z_{\text {Hod }}(f, s)$ into $Z_{\text {top }}(f, s)$, heuristically by taking the limit $u, v \rightarrow 1$.

We are especially interested in the poles of these zeta functions. For the topological zeta function the notion of pole is immediately clear; for the zeta function on the level of Hodge polynomials we say that a rational number $q$ is pole if $(u v)^{-q}$ is, considering $Z_{\text {Hod }}(f, s)$ as a rational function in the variable $T=(u v)^{-s}$. Being the only possible poles, we will call the elements of the set $\left\{-\frac{\nu_{i}}{N_{i}} \mid i \in T\right\}$ the candidate poles. So, to each irreducible component $E_{i}$ of $h^{-1}\left(f^{-1}\{0\}\right)$ we associate one candidate pole, namely the rational number $-\frac{\nu_{i}}{N_{i}}$. One should remark that for the (local) zeta functions $Z_{\text {Hod }}(f, s)$ and $Z_{\text {top }}(f, s)$ a candidate pole $s_{\circ}$ can only be a pole when there exists a component $E_{i}$ with $s_{\circ}=-\frac{\nu_{i}}{N_{i}}$ and satisfying $E_{i} \cap h^{-1}\{0\} \neq \emptyset$.

(1.4) Note. In what follows we will often suppose, without explicitly mentioning it anymore, that a polynomial $f \in \mathbb{C}\left[x_{1}, \ldots, x_{n}\right] \backslash \mathbb{C}$ and an embedded resolution $h: X \rightarrow \mathbb{A}_{\mathbb{C}}^{n}$ are given. Recall that we will also always assume that $f(0)=0$.

\section{The contribution of one exceptional component}

(2.1) Fix an exceptional variety $E$ with $h(E)=\{0\}$ and numerical data $(\nu, N)$. In this section we are interested in the contribution $\mathcal{R}$ of $E$ to the residue of $Z_{\text {Hod }}(f, s)$ at the candidate pole $-\frac{\nu}{N}$. We will find a condition which is sufficient to imply the non-vanishing of $\mathcal{R}$. We start with the following lemma, which is the main tool of the present paper. Essentially all the results on zeta functions will be corollaries of it.

(2.2) Lemma. Let $r \in \mathbb{Z}_{>0}$ and, for each $i \in\{1, \ldots, r\}$, let $\beta_{i} \in \mathbb{Z} \backslash\{0\}$. For each $I \subseteq\{1, \ldots, r\}$, let $p_{I}(x) \in \mathbb{C}[x]$ be a polynomial. Let $N, a, b \in \mathbb{Z}_{>0}$ with $a \in b \mathbb{Z}$. Then we 
have

$$
\left(\sum_{I \subseteq\{1, \ldots, r\}} p_{I}\left(x^{N}\right) \prod_{i \in I} \frac{x^{a N}-1}{x^{b \beta_{i}}-1}=0\right) \Rightarrow\left(\sum_{I \subseteq J} p_{I}\left(\xi_{b}\right) \prod_{i \in I} \frac{a N}{b \beta_{i}}=0\right) .
$$

Here we denote by $J$ the set $\left\{i \in\{1, \ldots, r\} \mid \beta_{i} \in N \mathbb{Z}\right\}$ and by $\xi_{b}$ a primitive $b$-th root of unity.

Proof. Denote by $R(x)$ the left-hand side of the implication in the statement above. One can easily show that there exists a primitive $(b N)$-th root of unity $\xi_{b N}$ such that $\xi_{b N}^{N}=\xi_{b}$. We will now exploit the relation

$$
R\left(\xi_{b N}\right)=0 .
$$

Of course $\xi_{b N}^{a N}=1$, and obviously $\xi_{b N}^{b \beta_{i}}=1$ if and only if $\beta_{i} \in N \mathbb{Z}$, thus if and only if $i \in J$. Fix $i \in J$ and denote $\left(x^{a N}-1\right) /\left(x^{b \beta_{i}}-1\right)$ by $T_{i}(x)$. By the rule of de l'Hôpital we then clearly have

$$
T_{i}\left(\xi_{b N}\right)=\frac{a N}{b \beta_{i}} .
$$

Since we now know that the evaluation of $\left(x^{a N}-1\right) /\left(x^{b \beta_{i}}-1\right)$ at $\xi_{b N}$ precisely does not vanish for $i \in J$ and that it equals $\frac{a N}{b \beta_{i}}$ in this case, we can conclude that

$$
\begin{aligned}
0 & =R\left(\xi_{b N}\right) \\
& =\sum_{I \subseteq J} p_{I}\left(\xi_{b}\right) \prod_{i \in I} \frac{a N}{b \beta_{i}}
\end{aligned}
$$

ending the proof of the lemma.

We are now ready to prove the main result of this section.

(2.3) Theorem. Let $f \in \mathbb{C}\left[x_{1}, \ldots, x_{n}\right] \backslash \mathbb{C}$. Fix an embedded resolution $h: X \rightarrow \mathbb{A}_{\mathbb{C}}^{n}$ of $f^{-1}\{0\}$ in $\mathbb{A}_{\mathbb{C}}^{n}$ and use the notation of Section 1. Let $E$ be an exceptional variety with $h(E)=\{0\}$ and numerical data $(\nu, N)$. Let $E_{i}, i=1, \ldots, r$, be the irreducible components of $h^{-1}\left(f^{-1}\{0\}\right)$, different from $E$, which intersect $E$. Suppose that $\alpha_{i}:=\nu_{i}-\frac{\nu}{N} N_{i} \neq 0$ for each $i \in\{1, \ldots, r\}$. Let $m \in \mathbb{Z}_{>0}$ and denote by $S_{m}$ the set $\left\{i \in\{1, \ldots, r\} \mid \alpha_{i} \in \frac{1}{m} \mathbb{Z}\right\}$. Then we have

$$
\left(\sum_{I \subseteq S_{m}} \frac{\chi\left(\left(E \cap E_{I}\right)^{\circ}\right)}{\prod_{i \in I} \alpha_{i}} \neq 0\right) \Rightarrow(\mathcal{R} \neq 0),
$$

where we denote by $\mathcal{R}$ the contribution of $E$ to the residue of $Z_{\mathrm{Hod}}(f, s)$ at $-\frac{\nu}{N}$.

Proof. Suppose that $\mathcal{R}=0$. Then, omitting some irrelevant factors from the expression for $\mathcal{R}$, we see that

$$
R:=\sum_{I \subseteq\{1, \ldots, r\}} H\left(\left(E \cap E_{I}\right)^{\circ}\right) \prod_{i \in I} \frac{u v-1}{(u v)^{\alpha_{i}}-1}=0 .
$$

Let $t \in \mathbb{Z}_{>0}$ be an arbitrary common denominator of the numbers $\alpha_{i}$. Then we obviously have

$$
t \alpha_{i} \in \mathbb{Z} \quad \text { for } i \in\{1, \ldots, r\} .
$$


Now we transform $R$ into a "normal" rational function $R_{x}$ in only one variable, say $x$. Here we mean by normal that the exponents occurring in $R_{x}$ are all integers. For this, we replace the variable $u$ by 1 and the variable $v$ by $x^{m t}$ in the expression for $R$. We obtain the rational function

$$
R_{x}:=\sum_{I \subseteq\{1, \ldots, r\}} H_{x}\left(\left(E \cap E_{I}\right)^{\circ}\right) \prod_{i \in I} \frac{x^{m t}-1}{x^{m t \alpha_{i}}-1},
$$

where $H_{x}\left(\left(E \cap E_{I}\right)^{\circ}\right)$ stands for $H\left(\left(E \cap E_{I}\right)^{\circ}\right)\left(1, x^{m t}\right)$. Because $R=0$, we also know that $R_{x}=0$. Now we apply Lemma 2.2 with $\beta_{i}=m t \alpha_{i}$ for $i \in\{1, \ldots, r\}, N=t, a=m, b=1$ and $p_{I}(x)=H\left(\left(E \cap E_{I}\right)^{\circ}\right)\left(1, x^{m}\right)$ for $I \subseteq\{1, \ldots, r\}$. We obtain that

$$
\begin{aligned}
0 & =\sum_{I \subseteq S_{m}} H\left(\left(E \cap E_{I}\right)^{\circ}\right)(1,1) \prod_{i \in I} \frac{m t}{m t \alpha_{i}} \\
& =\sum_{I \subseteq S_{m}} \frac{\chi\left(\left(E \cap E_{I}\right)^{\circ}\right)}{\prod_{i \in I} \alpha_{i}},
\end{aligned}
$$

which completes the proof of Theorem 2.3.

(2.4) Remarks.

(i) With the same notation as in Theorem 2.3 we also have the following implication:

$$
\left(\sum_{I \subseteq S_{m}} \frac{H\left(\left(E \cap E_{I}\right)^{\circ}\right)(-1,-1)}{\prod_{i \in I} \alpha_{i}} \neq 0\right) \Rightarrow(\mathcal{R} \neq 0),
$$

where in this case the integer $m$ has to be odd. To prove this we can proceed in the same way as in the previous proof. This time we replace both the variables $u$ and $v$ by $x^{m t}$. Applying Lemma 2.2 with $\beta_{i}=m t \alpha_{i}$ for $i \in\{1, \ldots, r\}, N=t, a=2 m, b=2$ and $p_{I}(x)=H\left(\left(E \cap E_{I}\right)^{\circ}\right)\left(x^{m}, x^{m}\right)$ for $I \subseteq\{1, \ldots, r\}$ will yield the implication.

(ii) We may drop the conditions that $h(E)=\{0\}$ and that $E$ is an exceptional variety, also allowing now that $E$ is an irreducible component of the strict transform of $f^{-1}\{0\}$, at least when we replace $\left(E \cap E_{I}\right)^{\circ}$ by $\left(E \cap E_{I}\right)^{\circ} \cap h^{-1}\{0\}$ in the statement. Also note that then we only have to suppose that $\alpha_{i} \neq 0$ for those components $E_{i}, i=1, \ldots, r$, which satisfy $E_{i} \cap h^{-1}\{0\} \neq \emptyset$.

We can also consider the contribution of $E$ to the residue of the function $Z_{\text {Hod,gl }}(f, s)$ at $-\frac{\nu}{N}$. Again we may omit the conditions that $h(E)=\{0\}$ and that $E$ is an exceptional variety, this time even without any change in the statement at all.

(iii) One can also associate a zeta function on the level of Hodge polynomials to an effective $\mathbb{Q}$-Cartier divisor $D$ on a $\mathbb{Q}$-Gorenstein variety with singular locus contained in Supp $D$ (in fact only the locus of log canonical singularities has to be contained in $\operatorname{Supp} D$ ), see [16]. It is remarkable that even in this general situation Theorem 2.3 still holds, and that we can still use the same easy proof as before.

(2.5) Example. Let $E=\mathbb{P}^{2}$ and let $F_{1}, F_{2}, F_{3}$ and $F_{4}$ be four rational curves on $E$ such that $\sum_{i=1}^{4} F_{i}$ has only normal crossings. Suppose that $d_{1}=d_{2}=d_{3}=1$ and that $d_{4}=2$, where $d_{i}$ stands for the degree of the curve $F_{i}$. 


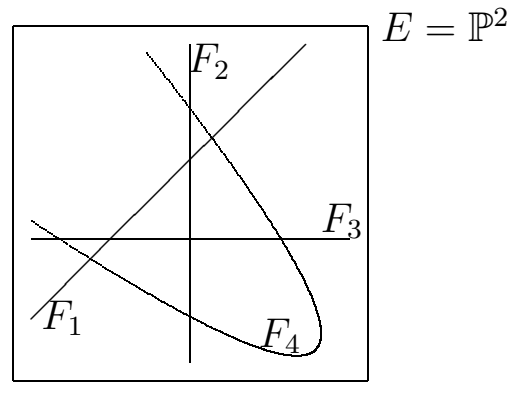

Figure 1

Let $\alpha_{1}=-1, \alpha_{2}=3 / 2, \alpha_{3}=1 / 4$ and $\alpha_{4}=5 / 8$. It is an interesting question on its own whether or not this configuration (together with the data $\alpha_{i}$ !) can occur as the intersection configuration in an embedded resolution. But here this does not matter because in the proof of Theorem 2.3 we did not use the presence of an embedded resolution in any way; indeed, we actually proved the implication for any given set of rational numbers $\left\{\alpha_{i} \mid 1 \leq i \leq r\right\}$ and for any (normal crossings) configuration $\sum_{i=1}^{r} F_{i}$ on a nonsingular variety $E$, where we then have to replace $\left(E \cap E_{I}\right)^{\circ}$ by $F_{I}^{\circ}$ and where we interpret $\mathcal{R}$ as the expression

$$
\sum_{I \subseteq\{1, \ldots, r\}} H\left(F_{I}^{\circ}\right) \prod_{i \in I} \frac{u v-1}{(u v)^{\alpha_{i}}-1} .
$$

Of course $F_{I}^{\circ}$ stands for $\cap_{i \in I} F_{i} \backslash \cup_{j \in\{1, \ldots, r\} \backslash I} F_{j}$; when $I=\emptyset$, we have $F_{\emptyset}^{\circ}=E^{\circ}=E \backslash$ $\cup_{i \in\{1, \ldots, r\}} F_{i}$.

Returning to the example, we obtain by Theorem 2.3 for $m=1$ that $\mathcal{R} \neq 0$, since

$$
\begin{aligned}
\sum_{I \subseteq\{1\}} \frac{\chi\left(F_{I}^{\circ}\right)}{\prod_{i \in I} \alpha_{i}} & =\chi\left(E^{\circ}\right)+\frac{\chi\left(F_{1}^{\circ}\right)}{\alpha_{1}} \\
& =4+2 \\
& \neq 0 .
\end{aligned}
$$

We should not expect the same theorem to be true for the topological zeta function $Z_{\text {top }}(f, s)$. Indeed, with the data of this example we have

$$
\begin{aligned}
\mathcal{R}_{\mathrm{top}} & :=\sum_{I \subseteq\{1,2,3,4\}} \chi\left(F_{I}^{\circ}\right) \prod_{i \in I} \frac{1}{\alpha_{i}} \\
& =4-2\left(\sum_{i=1}^{3} \frac{1}{\alpha_{i}}\right)-\frac{4}{\alpha_{4}}+\frac{2}{\alpha_{4}}\left(\sum_{i=1}^{3} \frac{1}{\alpha_{i}}\right)+\frac{1}{\alpha_{1} \alpha_{2}}+\frac{1}{\alpha_{1} \alpha_{3}}+\frac{1}{\alpha_{2} \alpha_{3}} \\
& =0 .
\end{aligned}
$$

(2.6) Comments concerning the case $n=3$.

For the remarks in this subsection we fix an embedded resolution $h: X \rightarrow \mathbb{A}_{\mathbb{C}}^{3}$ of $f^{-1}\{0\}$ in $\mathbb{A}_{\mathbb{C}}^{3}$ in the sense of Hironaka's Main Theorem II [5, p. 142] by means of blowing-ups. 
(i) In [10, Theorem 3.4] we proved the following implication when $E$ is non-rational and $\alpha_{i} \neq 1$ for $i \in\{1, \ldots, r\}$ :

$$
\left(\bar{\kappa}\left(E^{\circ}\right)=2\right) \Rightarrow(\mathcal{R} \neq 0) .
$$

We also know by [4, Proposition 2] that $E^{\circ}$ having maximal logarithmic Kodaira dimension implies that $\chi\left(E^{\circ}\right)>0$ when $\cup_{i \in\{1, \ldots, r\}}\left(E \cap E_{I}\right)$ is connected.

So, combining the latter fact with Theorem 2.3, we see that when $\cup_{i \in\{1, \ldots, r\}}\left(E \cap E_{I}\right)$ is connected and none of the numbers $\alpha_{i}$ is an integer, the condition on $E$ of being non-rational may be dropped in the statement of [10, Theorem 3.4].

(ii) The combination of Theorem 2.3 and Remark 2.4(i) suggests that the contribution $\mathcal{R}$ to the residue does not vanish, unless certain conditions on the genera are satisfied. We will show by combining these two facts that the vanishing of $\mathcal{R}$ implies that

$$
2 g=\sum_{i=1}^{r} g_{i}-\sum_{\left\{i \mid \alpha_{i} \in \mathbb{Z}\right\}} \frac{g_{i}}{\alpha_{i}}
$$

where we denote by $g_{i}, i=1, \ldots, r$, the genera of the irreducible components $F_{i}$ of the intersections $E \cap E_{k}, k \in T$, where $E_{k}$ is another exceptional surface or an irreducible component of the strict transform of $f^{-1}\{0\}$. The number $g$ has to be interpreted as follows. When $E$ is created as a ruled surface $E_{0}$ over a curve $D$, we denote by $g$ the genus of $D$; when $E$ is created by a point-centered blowing-up we define $g$ to be zero.

Indeed, when $\mathcal{R}=0$ we have

$$
\begin{cases}\chi\left(E^{\circ}\right)+\sum_{i \in S} \frac{\chi\left(F_{i}^{\circ}\right)}{\alpha_{i}}+\sum_{\{i, j\} \subseteq S} \frac{\chi\left(F_{i} \cap F_{j}\right)}{\alpha_{i} \alpha_{j}} & =0 \\ H\left(E^{\circ}\right)(-1,-1)+\sum_{i \in S} \frac{H\left(F_{i}^{\circ}\right)(-1,-1)}{\alpha_{i}}+\sum_{\{i, j\} \subseteq S} \frac{H\left(F_{i} \cap F_{j}\right)(-1,-1)}{\alpha_{i} \alpha_{j}}=0,\end{cases}
$$

where we denote by $S$ the set $\left\{i \in\{1, \ldots, r\} \mid \alpha_{i} \in \mathbb{Z}\right\}$. Because clearly

$$
\frac{\chi\left(F_{i} \cap F_{j}\right)}{\alpha_{i} \alpha_{j}}=\frac{H\left(F_{i} \cap F_{j}\right)(-1,-1)}{\alpha_{i} \alpha_{j}},
$$

we obtain by an easy calculation, see for example [10, Lemma 2.2], that

$$
\begin{aligned}
0 & =\chi\left(E^{\circ}\right)-H\left(E^{\circ}\right)(-1,-1)+\sum_{i \in S} \frac{\chi\left(F_{i}^{\circ}\right)-H\left(F_{i}^{\circ}\right)(-1,-1)}{\alpha_{i}} \\
& =-8 g+\sum_{i=1}^{r} 4 g_{i}+\sum_{i \in S} \frac{-4 g_{i}}{\alpha_{i}}
\end{aligned}
$$

proving the equality we are after. One should remark that this fact is not new, since it also immediately follows from [10, Proposition 2.5]. There however we needed very long and laborious calculations.

(2.7) Example. Suppose again that the embedded resolution $h: X \rightarrow \mathbb{A}_{\mathbb{C}}^{n}$ consists of a composition of blowing-ups. Before discussing the example we state the following special 
case of a result of Veys [12, Theorem 6.2]. When E is created by a blowing-up $\pi$ at a closed subvariety $D$ and when the following blowing-ups do not "change" it anymore, then we have

$$
\sum_{i=1}^{r} d_{i}\left(\alpha_{i}-1\right)=\operatorname{dim} D-n,
$$

where $d_{i}, i=1, \ldots, r$, is the degree of the intersection cycle $F_{i} \cdot F$ on $F$ for any fiber $F \cong \mathbb{P}^{n-\operatorname{dim} D-1}$ of $\pi_{\mid E}: E \rightarrow D$ over a point of $D$. As before we denote here by $F_{i}$ the irreducible components of the intersections $E \cap E_{k}, k \in T$, where $E_{k}$ is another exceptional variety or an irreducible component of the strict transform of $f^{-1}\{0\}$.

By "the following blowing-ups do not change $E$ anymore" we mean that the composition $\tilde{h}$ of the blowing-ups which still have to be performed after $\pi$ yields an isomorphism between the strict transform of $E$ under $\tilde{h}$ and $E$ itself. Obviously this is equivalent to the condition that each of the centers of these blowing-ups is either disjoint with $E$ or a subvariety of $E$ of codimension 1 in $E$.

Let $n=4$, suppose that $E$ is created by a point-centered blowing-up and that it did not "change" anymore after its creation. Hence $E=\mathbb{P}^{3}$. Suppose furthermore that there is only one intersection surface $F_{1}$ on $E$ and that it is the nonsingular quadric surface. By the above result of Veys we see that $\alpha_{1}=-1$. In this example we have

$$
\chi\left(E^{\circ}\right)=4-4=0 .
$$

But the contribution $\mathcal{R}$ to the residue does not vanish. Indeed,

$$
\begin{aligned}
\mathcal{R} & =c\left((u v)^{3}+(u v)^{2}+u v+1-\left((u v)^{2}+2 u v+1\right)+\left((u v)^{2}+2 u v+1\right) \frac{u v-1}{(u v)^{\alpha_{1}}-1}\right) \\
& =-2 c u v(1+u v) \\
& \neq 0
\end{aligned}
$$

where $c$ is a nonzero factor. In [1, Conjecture 1.2.2] Denef and Jacobs formulate the conjecture that, under certain conditions, the vanishing of $\chi\left(E^{\circ}\right)$ will imply the vanishing of (the $p$-adic analogue of) $\mathcal{R}$. One of these conditions says that none of the numbers $\alpha_{i}$ is an integer. In a way Theorem 2.3 explains how the existence of integral numbers $\alpha_{i}$ can affect the situation. Indeed, since $\alpha_{1} \in \mathbb{Z}$, our result tells us that in this situation we don't have to consider $\chi\left(E^{\circ}\right)$, but rather $\chi\left(E^{\circ}\right)+\chi\left(F_{1}^{\circ}\right) / \alpha_{1}$. And this new expression obviously does not vanish in this case.

\section{Candidate poles of order 1}

(3.1) In this section we will see that our new technique also enables us to add the several contributions to the residue at one fixed rational number $s_{\circ}$, providing a sufficient condition for $s_{\circ}$ being a pole (of order 1 ) of the zeta function on the level of Hodge polynomials.

(3.2) Theorem. Let $f \in \mathbb{C}\left[x_{1}, \ldots, x_{n}\right] \backslash \mathbb{C}$ and let $s_{\circ} \in \mathbb{Q}$. Fix an embedded resolution $h: X \rightarrow \mathbb{A}_{\mathbb{C}}^{n}$ of $f^{-1}\{0\}$ in $\mathbb{A}_{\mathbb{C}}^{n}$ and use the notation of Section 1 . Let $E_{j}, j=1, \ldots, k$, be precisely the irreducible components of $h^{-1}\left(f^{-1}\{0\}\right)$ satisfying $s_{\circ}=-\frac{\nu_{j}}{N_{j}}$. Let $E_{i}^{(j)}$, 
$i=1, \ldots, r_{j}$, be the irreducible components of $h^{-1}\left(f^{-1}\{0\}\right)$, different from $E_{j}$, which intersect $E_{j}$. Suppose that $\alpha_{i}^{(j)}:=\nu_{i}^{(j)}+s_{\circ} N_{i}^{(j)} \neq 0$, for each $j \in\{1, \ldots, k\}$ and $i \in\left\{1, \ldots, r_{j}\right\}$ satisfying $E_{j} \cap E_{i}^{(j)} \cap h^{-1}\{0\} \neq \emptyset$. Denote by $S^{(j)}$ the set $\left\{i \in\left\{1, \ldots, r_{j}\right\} \mid \alpha_{i}^{(j)} \in \mathbb{Z}\right\}$.

Then we have the following. If

$$
\sum_{j=1}^{k} \frac{1}{N_{j}}\left(\sum_{\substack{I \subseteq S^{(j)} \\\left(E_{j} \cap E_{I}^{(j)}\right)^{\circ} \cap h^{-1}\{0\} \neq \emptyset}} \frac{\chi\left(\left(E_{j} \cap E_{I}^{(j)}\right)^{\circ} \cap h^{-1}\{0\}\right)}{\prod_{i \in I} \alpha_{i}^{(j)}}\right) \neq 0,
$$

then $s_{\circ}$ is a pole (of order 1 ) of $Z_{\mathrm{Hod}}(f, s)$.

Proof. The total residue of $Z_{\mathrm{Hod}}(f, s)$ at $s_{\circ}$ is

$$
\sum_{j=1}^{k} \sum_{\substack{I \subseteq\left\{1, \ldots, r_{j}\right\} \\\left(E_{j} \cap E_{I}^{(j)}\right)^{\circ} \cap h^{-1}\{0\} \neq \emptyset}} H\left(\left(E_{j} \cap E_{I}^{(j)}\right)^{\circ} \cap h^{-1}\{0\}\right) \frac{1-u v}{N_{j}(u v)^{s_{\circ}}} \prod_{i \in I} \frac{u v-1}{(u v)^{\alpha_{i}^{(j)}}-1} .
$$

Now we can use exactly the same argument as in the proof of Theorem 2.3 (for $m=1$ ).

(3.2.1) Remarks.

(i) Following the details of the proof of Remark 2.4(i), instead of those of the proof of Theorem 2.3, we obtain another variant of Theorem 3.2. For the statement of this version we just have to replace

$$
\chi\left(\left(E_{j} \cap E_{I}^{(j)}\right)^{\circ} \cap h^{-1}\{0\}\right) \text { by } H\left(\left(E_{j} \cap E_{I}^{(j)}\right)^{\circ} \cap h^{-1}\{0\}\right)(-1,-1) .
$$

(ii) Theorem 3.2 also holds for the global zeta function $Z_{\text {Hod,gl }}(f, s)$, when we replace $h^{-1}\{0\}$ by $X$ everywhere in the statement.

(3.3) In the next theorem we will additionally assume that none of the numbers $\alpha_{i}^{(j)}$ is an integer. This will be sufficient, except for a minor and very plausible assumption on the embedded resolution $h$, in order to obtain that $s_{\circ}$ is always a pole of $Z_{\text {Hod }}(f, s)$ whenever there exists an exceptional variety $E_{j}$ with $s_{\circ}=-\frac{\nu_{j}}{N_{j}}, h\left(E_{j}\right)=\{0\}$ and $\chi\left(E_{j}^{\circ}\right) \neq 0$. We first need the following lemma, which is a special case of [14, Theorem 3.5].

Lemma. Let $E_{j}$ be an exceptional hypersurface with $h\left(E_{j}\right)=\{0\}$ and let $d \in \mathbb{Z}_{>1}$. Suppose that $d \mid N_{j}$ and that $d \nmid N_{i}$ for all other components $E_{i}$ of $h^{-1}\left(f^{-1}\{0\}\right)$ having nonempty intersection with $E_{j}$. Then

$$
(-1)^{n-1} \chi\left(E_{j}^{\circ}\right) \geq 0
$$

(3.4) Theorem. We use the notation of Theorem 3.2, but we suppose here that $\alpha_{i}^{(j)} \notin \mathbb{Z}$ for each $j \in\{1, \ldots, k\}$ and $i \in\left\{1, \ldots, r_{j}\right\}$ satisfying $E_{j} \cap E_{i}^{(j)} \cap h^{-1}\{0\} \neq \emptyset$. Furthermore 
we suppose for each $j \in\{1, \ldots, k\}$ that either $E_{j}^{\circ} \cap h^{-1}\{0\}=\emptyset$ or $E_{j}^{\circ} \subseteq h^{-1}\{0\}$.

If there exists an exceptional variety $E_{j}$ satisfying $j \in\{1, \ldots, k\}, E_{j} \subseteq h^{-1}\{0\}$ and $\chi\left(E_{j}^{\circ}\right) \neq$ 0 , then $s_{\circ}$ is a pole of $Z_{\mathrm{Hod}}(f, s)$.

Remark. Obviously the condition that either $E_{j}^{\circ} \cap h^{-1}\{0\}=\emptyset$ or $E_{j}^{\circ} \subseteq h^{-1}\{0\}$ is satisfied when $h^{-1}\{0\}=\bigcup_{j \in J^{\prime}} E_{j}$ for some subset $J^{\prime}$ of $T$. But this last condition is of course fulfilled when we consider an embedded resolution $h$ constructed by first blowing up $\mathbb{A}_{\mathbb{C}}^{n}$ at the origin, whatever the situation might be (and then for example just following Hironaka's construction). So, this extra condition in the statement of Theorem 3.4 only means that we have to consider a well-chosen embedded resolution, rather than that we are excluding some polynomials $f$ and hence zeta functions $Z_{\text {Hod }}(f, s)$.

Proof. By Theorem 3.2 we only have to prove that

$$
\sum_{j \in J} \frac{1}{N_{j}} \chi\left(E_{j}^{\circ}\right) \neq 0,
$$

where we denote by $J$ the set $\left\{j \in\{1, \ldots, k\} \mid h\left(E_{j}\right)=\{0\}\right\}$. Note that we use here the extra condition on our embedded resolution. Write

$$
s_{\circ}=-\frac{a}{d},
$$

where $a$ and $d$ are two natural numbers which are relatively prime. With this choice for $d$ all the assumptions of Lemma 3.3 are fulfilled for any $E_{j}$ with $j \in J$, by the conditions on the numbers $\alpha_{i}^{(j)}$, since by Zariski's Main Theorem there is for each exceptional variety $E_{j}$ at least one intersecting component $E_{i}^{(j)}$. This implies that the terms in $(*)$ are either all positive or all negative. Now we are done, since at least one of those terms is supposed to be nonzero.

\section{(3.4.1) Remarks.}

(i) When each of the components $E_{j}$, with $j \in\{1, \ldots, k\}$ and $\chi\left(E_{j}^{\circ}\right) \neq 0$, is entirely mapped onto one point by the resolution $h$, then the analogue of Theorem 3.4 for $Z_{\text {top,gl }}(f, s)$, obtained by replacing $h^{-1}\{0\}$ by $X$ in its statement, is also true.

(ii) As in Remark 2.4(iii), it is interesting to note that in fact Theorem 3.2 and Theorem 3.4 even hold for the zeta functions associated to an effective $\mathbb{Q}$-Cartier divisor $D$ on a $\mathbb{Q}$-Gorenstein variety with singular locus contained in $\operatorname{Supp} D$. For this we need Lemma 3.3 also to be true in the singular case; see the proof of [15, Theorem 3.3].

(3.5) The results of Section 2 and Section 3 are also true for the well-known motivic zeta function, see for example [3, Section 2] for the definition of this function. They are immediate corollaries of the corresponding results for the Hodge zeta function by using a specialization argument. For the notion of a pole of the motivic zeta function we refer to [11, Definition 4.7].

(3.6) In this subsection we briefly consider the situation of candidate poles of higher order. To each element of the partition $\left\{E_{I}^{\circ} \mid I \subseteq T\right.$ and $\left.E_{I}^{\circ} \neq \emptyset\right\}$ of $X$ we associate a number of 
candidate poles, all provided with a certain order. Indeed, we will say that $E_{I}^{\circ}$ induces the candidate pole $s_{\circ}$ of order $r$ if $E_{I}^{\circ} \neq \emptyset$ and the cardinality of the set $\left\{i \in I \mid s_{\circ}=-\frac{\nu_{i}}{N_{i}}\right\}$ is precisely $r$. We say that $E_{I}^{\circ}$ strictly induces the candidate pole $s_{\circ}$ of order $r$ if moreover $|I|=r$, or equivalently $\operatorname{dim} E_{I}^{\circ}=n-r$.

Then the following analogue of Theorem 3.2 can easily be obtained by applying a very similar argument.

(3.6.1) Theorem. Let $f \in \mathbb{C}\left[x_{1}, \ldots, x_{n}\right] \backslash \mathbb{C}$ and let $s_{\circ} \in \mathbb{Q}$. Fix an embedded resolution $h: X \rightarrow \mathbb{A}_{\mathbb{C}}^{n}$ of $f^{-1}\{0\}$ in $\mathbb{A}_{\mathbb{C}}^{n}$ and use the notation of Section 1 . Let $I_{j}, j=1, \ldots, k$, be precisely the subsets $I$ of $T$ such that $E_{I}^{\circ}$ strictly induces $s_{\circ}$ as a candidate pole of order $r$. Let $E_{i}^{(j)}, i=1, \ldots, r_{j}$, be the irreducible components of $h^{-1}\left(f^{-1}\{0\}\right)$, different from $E_{l}$ for $l \in I_{j}$, which intersect $E_{I_{j}}$. Suppose that $\alpha_{i}^{(j)}:=\nu_{i}^{(j)}+s_{\circ} N_{i}^{(j)} \neq 0$, for each $j \in\{1, \ldots, k\}$ and $i \in\left\{1, \ldots, r_{j}\right\}$ satisfying $E_{I_{j}} \cap E_{i}^{(j)} \cap h^{-1}\{0\} \neq \emptyset$. Denote by $S^{(j)}$ the set $\left\{i \in\left\{1, \ldots, r_{j}\right\} \mid \alpha_{i}^{(j)} \in \mathbb{Z}\right\}$.

Then we have the following. If

$$
\sum_{j=1}^{k} \frac{1}{\prod_{l \in I_{j}} N_{l}}\left(\sum_{\substack{I \subseteq S^{(j)} \\\left(E_{I_{j}} \cap E_{I}^{(j)}\right)^{\circ} \cap h^{-1}\{0\} \neq \emptyset}} \frac{\chi\left(\left(E_{I_{j}} \cap E_{I}^{(j)}\right)^{\circ} \cap h^{-1}\{0\}\right)}{\prod_{i \in I} \alpha_{i}^{(j)}}\right) \neq 0,
$$

then $s_{\circ}$ is a pole of order $r$ of $Z_{\mathrm{Hod}}(f, s)$.

We will now give in a way the analogue of Theorem 3.4 for poles of higher order. We first need the following definition. Fix $d \in \mathbb{Z}_{>1}$. We say that $E_{I}, I \subseteq T$, satisfies the affineness condition for $d$ if $E_{I}$ is complete, $d$ divides all $N_{i}$ with $i \in I$ and $E_{I} \backslash \bigcup_{d \nmid N_{j}} E j$ is affine; see [14, Subsection 4.1] for this definition in the case that $|I|=1$. Then the analogue of Theorem 3.4 is as follows.

(3.6.2) Theorem. We use the notation of Theorem 3.6.1, but we suppose here that $\alpha_{i}^{(j)} \notin \mathbb{Z}$ for each $j \in\{1, \ldots, k\}$ and $i \in\left\{1, \ldots, r_{j}\right\}$ satisfying $E_{I_{j}} \cap E_{i}^{(j)} \cap h^{-1}\{0\} \neq \emptyset$, and that for each $j \in\{1, \ldots, k\}$ either $E_{I_{j}}^{\circ} \cap h^{-1}\{0\}=\emptyset$ or $E_{I_{j}}^{\circ} \subseteq h^{-1}\{0\}$. Also suppose that for each $j \in\{1, \ldots, k\}$ with $E_{I_{j}} \subseteq h^{-1}\{0\}$ and $\chi\left(E_{I_{j}}^{\circ}\right) \neq 0$ there exists an integer $d_{j}$ such that $E_{I_{j}}$ satisfies the affineness condition for $d_{j}$.

If there exists an element $j \in\{1, \ldots, k\}$ such that $E_{I_{j}} \subseteq h^{-1}\{0\}$ and $\chi\left(E_{I_{j}}^{\circ}\right) \neq 0$, then $s_{\circ}$ is a pole of order $r$ of $Z_{\mathrm{Hod}}(f, s)$.

The proof of Theorem 3.6.2 mainly depends on the following lemma, playing in this case the role which Lemma 3.3 played in the proof of Theorem 3.4. It can be proved using exactly the same arguments as in the proof of [14, Proposition 4.3(i)], where Veys obtained the same conclusion but under slightly different conditions.

(3.6.3) Lemma. Fix $d \in \mathbb{Z}_{>1}$. Let $I \subseteq T$ such that $E_{I}$ satisfies the affineness condition for d. Then

$$
(-1)^{n-|I|} \chi\left(E_{I}^{\circ}\right) \geq 0
$$


Theorem 3.6.1 and Theorem 3.6.2 also hold for the zeta functions associated to an effective $\mathbb{Q}$-Cartier divisor $D$ on a $\mathbb{Q}$-Gorenstein variety with singular locus contained in Supp $D$.

For more details about this subsection we refer to [8, Section 6.6].

\section{Excursion to the situation of curves}

(4.1) Suppose in this section that $n=2$. Then the irreducible components of $h^{-1}\left(f^{-1}\{0\}\right)$ are all curves. In [13, Theorem 4.3] Veys already gave for this situation a complete geometric determination of the poles of $Z_{\mathrm{top}}(f, s)$, see also Theorem 0.2.1. We will see that our new technique enables us to give an easy proof of this fact for $Z_{\mathrm{Hod}}(f, s)$. Of course this result for $Z_{\text {Hod }}(f, s)$ also immediately follows from the version of Veys for $Z_{\text {top }}(f, s)$. We start with the following lemma.

(4.2) Lemma. Let $k \in \mathbb{Z}_{\geq 0}$ and let $\alpha_{1}, \ldots, \alpha_{k}$ be $k$ nonzero rational numbers satisfying $\sum_{i=1}^{k} \alpha_{i}=k-2$. Then

$$
\mathcal{R}:=u v+1-k+\sum_{i=1}^{k} \frac{u v-1}{(u v)^{\alpha_{i}}-1}=0
$$

if and only if precisely one or two of the numbers $\alpha_{i}$ are different from 1.

Remark. Let $E$ be an exceptional curve satisfying $h(E)=\{0\}$ and intersecting other components $E_{i}$ exactly $k$ times. Suppose that $\alpha_{i}:=\nu_{i}-\frac{\nu}{N} N_{i} \neq 0$ for each $i \in\{1, \ldots, k\}$. Then, up to some irrelevant nonzero factor, the expression $\mathcal{R}$ is the contribution of $E$ to the residue of $Z_{\text {Hod }}(f, s)$ at $-\frac{\nu}{N}$. The relation $\sum_{i=1}^{k} \alpha_{i}=k-2$ between the rational numbers $\alpha_{i}$ is precisely the formula of Veys mentioned in Example 2.7.

Proof. Obviously we may suppose that $\alpha_{i} \neq 1$ for all $i \in\{1, \ldots, k\}$. Then we have to prove that $\mathcal{R}=0$ if and only if $k=1$ or 2 . When $k=1$ or 2 an easy calculation shows that the vanishing of $\mathcal{R}$ is implied by the condition $\sum_{i=1}^{k} \alpha_{i}=k-2$.

On the other hand one can prove in exactly the same way as in the proof of Theorem 2.3 (here with $m=1$ ) that if

$$
2-k+\sum_{i=1}^{r} \frac{1}{\alpha_{i}} \neq 0
$$

where $\alpha_{1}, \ldots, \alpha_{r}$ are exactly the integers among the numbers $\alpha_{i}$, then $\mathcal{R} \neq 0$. Since $k>0$ by the condition of the lemma, it is sufficient to show that $k \geq 3$ implies that $2-k+\sum_{i=1}^{r} \frac{1}{\alpha_{i}} \neq 0$. Suppose that $k \geq 3$. We distinguish two possibilities; either $r \leq k-2$ or $r=k$. Remark that $r=k-1$ is impossible by the condition $\sum_{i=1}^{k} \alpha_{i}=k-2$. First consider the case $r \leq k-2$. Then

$$
\sum_{i=1}^{r} \frac{1}{\alpha_{i}} \leq \frac{r}{2} \leq \frac{k-2}{2}<k-2
$$

where the last inequality is true since $k \geq 3$. Now we treat the case $r=k$. Then at least one of the numbers $\alpha_{i}$ is negative, say $\alpha_{1}$ for example; this easily follows from the condition $\sum_{i=1}^{k} \alpha_{i}=k-2$. We thus have

$$
\sum_{i=1}^{k} \frac{1}{\alpha_{i}}=\frac{1}{\alpha_{1}}+\sum_{i=2}^{k} \frac{1}{\alpha_{i}}<\frac{k-1}{2} \leq k-2,
$$


where again the last inequality holds since $k \geq 3$. This completes the proof of Lemma 4.2 .

(4.2.1) Remarks.

(i) It is worth mentioning that we do not assume any upper bound at all on the absolute values $\left|\alpha_{i}\right|$ of the numbers $\alpha_{i}$. This contrasts sharply with the arguments in [13], which intensively use the existence of such an upper bound.

(ii) Rereading the proof of the lemma, we notice that we actually proved that $2-k+$ $\sum_{i=1}^{r} \frac{1}{\alpha_{i}}<0$ whenever $\sum_{i=1}^{k} \alpha_{i}=k-2$ and at least three of the numbers $\alpha_{i}$ are different from 1. This remark will enable us to control the addition of the contributions (to the residue at a candidate pole of order 1 ) of several exceptional curves, see the proof of Proposition 4.3.

We will now prove the geometric determination of the poles of $Z_{\mathrm{Hod}}(f, s)$.

(4.3) Proposition. Let $f \in \mathbb{C}\left[x_{1}, x_{2}\right] \backslash \mathbb{C}$ and let $s_{\circ} \in \mathbb{Q}$. Fix an (arbitrary) embedded resolution $h: X \rightarrow \mathbb{A}_{\mathbb{C}}^{2}$ of $f^{-1}\{0\}$ in $\mathbb{A}_{\mathbb{C}}^{2}$ and use the notation of Section 1 . Then $s_{\circ}$ is a pole of $Z_{\mathrm{Hod}}(f, s)$ if and only if

(i) $s_{\circ}=-\frac{1}{N_{i}}$ for some irreducible component $E_{i}$ of the strict transform of $f^{-1}\{0\}$ satisfying $0 \in h\left(E_{i}\right)$ or

(ii) $s_{\circ}=-\frac{\nu_{i}}{N_{i}}$ for some exceptional curve $E_{i}$ satisfying $h\left(E_{i}\right)=\{0\}$ and intersecting other components $E_{j}$ with $\nu_{j}-\frac{\nu_{i}}{N_{i}} N_{j} \neq 1$ at least three times.

Proof. Suppose first that $s_{\circ}$ is a pole of order 2 of $Z_{\text {Hod }}(f, s)$. Clearly this is equivalent to the existence of intersecting components $E_{i}$ and $E_{j}$ of $h^{-1}\left(f^{-1}\{0\}\right)$ satisfying $s_{\circ}=-\frac{\nu_{i}}{N_{i}}=-\frac{\nu_{j}}{N_{j}}$ and $E_{\{i, j\}} \cap h^{-1}\{0\} \neq \emptyset$. If condition (i) is not fulfilled, one easily verifies by using (repeatedly) the relation of Veys, stated in Example 2.7, and by combinig this with the finiteness of the resolution graph that condition (ii) has to be satisfied; see [11, Proof of Theorem 3.4] for the details of this argumentation.

When $s_{\circ}$ is a pole of order 1 we are done by Lemma 4.2, recall the remark under the statement of Lemma 4.2.

Suppose that condition (i) or (ii) is fulfilled. From now on we exclude the trivial cases where the germ $(f, 0)$ is analytically equivalent to $\left(x_{1}^{N}, 0\right)$ or $\left(x_{1}^{N} x_{2}^{N^{\prime}}, 0\right)$ for some $N, N^{\prime} \in \mathbb{Z}_{>0}$, see also [13, Section 3]. We may also assume that there do not exist intersecting components $E_{i}$ and $E_{j}$ of $h^{-1}\left(f^{-1}\{0\}\right)$ satisfying $s_{\circ}=-\frac{\nu_{i}}{N_{i}}=-\frac{\nu_{j}}{N_{j}}$ and $h\left(E_{i} \cap E_{j}\right)=\{0\}$, since otherwise we obviously have a pole of order 2 . Now we are in the situation of Theorem 3.2. Let $k$ be the number of exceptional curves $E_{i}$ satisfying $s_{\circ}=-\frac{\nu_{i}}{N_{i}}$ and $h\left(E_{i}\right)=\{0\}$ and intersecting other components $E_{j}$ with $\nu_{j}-\frac{\nu_{i}}{N_{i}} N_{j} \neq 1$ at least three times.

First suppose that $k \geq 1$. Studying the crucial expression in Theorem 3.2 and combining it with Remark 4.2.1(ii), we see that it is sufficient to prove that

$$
\alpha_{i}:=\nu_{i}+s_{\circ} N_{i}<0,
$$


where $E_{i}$ is an exceptional curve intersecting an irreducible component $E_{j}$ of the strict transform of $f^{-1}\{0\}$ satisfying $s_{\circ}=-\frac{1}{N_{j}}$ and $h\left(E_{i} \cap E_{j}\right)=\{0\}$. This is implied by [13, Subsection 3.12], since $\alpha_{i} \neq 0$ and $k \geq 1$.

When $k=0$ a straightforward calculation will still show that the residue of $Z_{\text {Hod }}(f, s)$ at $s_{\circ}$ is nonzero. The main point is that the numbers $\alpha_{i}$ in $(*)$ are either all positive or all negative. They will be positive precisely in the case when $\left|s_{\circ}\right|$ is the minimum of the set of all absolute values of the candidate poles of $Z_{\text {Hod }}(f, s)$. Remark that in fact there is only one number $\alpha_{i}$ in this case, see [13, Subsection 3.12].

(4.3.1) Remarks.

(i) When one applies this proposition using the minimal embedded resolution, it should be clear that the condition "with $\nu_{j}-\frac{\nu_{i}}{N_{i}} N_{j} \neq 1$ " can be omitted, see $[7$, Proposition II.3.1] or [11, Lemma 2.6].

(ii) Using Remark 4.2.1(ii) in the same way as in the previous proof, we can provide another argumentation for the hardest part of the proof of [11, Theorem 3.4]. There we gave a complete geometric determination of the poles of the Hodge zeta functions associated to a regular function on a normal surface germ.

\section{References}

[1] J. Denef and P. Jacobs, On the vanishing of principal value integrals, Comptes Rendus de l'Académie des Sciences de Paris 326 (1998), 1041-1046.

[2] J. Denef and F. Loeser, Caractéristiques d'Euler-Poincaré, fonctions zeta locales, et modifications analytiques, J. Amer. Math. Soc. 5 (1992), 705-720.

[3] Motivic Igusa zeta functions, J. Algebraic Geom. 7 (1998), 505-537.

[4] R. V. Gurjar and A. J. Parameswaran, Open surfaces with non-positive Euler characteristic, Compositio Math. 99 (1995), 213-229.

[5] H. Hironaka, Resolution of an algebraic variety over a field of characteristic zero, Ann. of Math. 79 (1964), 109-326.

[6] J. Kollár, Singularities of Pairs, Summer Research Institute on Algebraic Geometry (Santa Cruz 1995), Proceedings of Symposia in Pure Mathematics 62.1 (Amer. Math. Soc., 1997), 221-287.

[7] F. Loeser, Fonctions d'Igusa p-adiques et polynômes de Bernstein, Amer. J. Math. 110 (1988), 1-22.

[8] B. Rodrigues, Geometric determination of the poles of motivic and topological zeta functions, Ph. D. thesis, University Leuven, 2002, available at http://www.wis.kuleuven.ac.be/algebra/artikels/artikelse.htm.

[9] _ Geometric determination of the poles of highest and second highest order of Hodge and motivic zeta functions, preprint (2003). 
[10] Logarithmic Kodaira dimension and the poles of the Hodge and motivic zeta functions for surfaces, Manuscripta Math. 112 (2003), 137-159.

[11] B. Rodrigues and W. Veys, Poles of zeta functions on normal surfaces, Proc. London Math. Soc. 87 (2003), 164-196.

[12] W. Veys, Relations between numerical data of an embedded resolution, Amer. J. Math. 113 (1991), 573-592.

[13] Determination of the poles of the topological zeta function for curves, Manuscripta Math. 87 (1995), 435-448.

[14] On Euler characteristics associated to exceptional divisors, Trans. Amer. Math. Soc. 347 (1995), 3287-3300.

[15] - The topological zeta function associated to a function on a normal surface germ, Topology 38 (1999), 439-456.

[16] Z Z Z Z functions and 'Kontsevich invariants' on singular varieties, Canad. J. Math. 53 (2001), 834-865.

K.U.LEUVEN, Departement Wiskunde, Celestijnenlaan 200B, 3001 LEUVEN, BELGIUM E-mail address: bart.rodrigues@wis.kuleuven.ac.be 